\title{
Erratum to: Semigroups of max-plus linear operators
}

\author{
Boris Andreianov ${ }^{1}$ • Marjeta Kramar Fijavž2,3 \\ Aljoša Peperko $^{3,4}$. Eszter Sikolya ${ }^{5}$
}

Received: 12 May 2016 / Accepted: 15 May 2016/ Published online: 4 April 2017

(C) Springer Science+Business Media New York 2017

\section{Erratum to: Semigroup Forum DOI 10.1007/s00233-015-9761-x}

Boris Andreianov determined that the examples of max-additive and max-plus linear semigroups in the last section of the article [6] are given inaccurately, i.e., [6, Proposition 4.1] is not true as stated and [6, Proposition 4.2] does not hold without some additional assumptions. Jointly we are able to correct the issues as follows.

Communicated by Markus Haase.

The online version of the original article can be found under doi:10.1007/s00233-015-9761-X.

$凶$ Marjeta Kramar Fijavž

marjeta.kramar@fgg.uni-lj.si

Boris Andreianov

boris.andreianov@1mpt.univ-tours.fr

Aljoša Peperko

aljosa.peperko@fs.uni-lj.si

Eszter Sikolya

seszter@cs.elte.hu

1 LMPT CNRS UMR7350, Université de Tours, Parc Grandmont, 37200 Tours, France

2 Faculty of Civil and Geodetic Engineering, University of Ljubljana, Jamova 2, 1000 Ljubljana, Slovenia

3 Institute of Mathematics, Physics, and Mechanics, Jadranska 19, 1000 Ljubljana, Slovenia

4 Faculty of Mechanical Engineering, University of Ljubljana, Aškerčeva 6, 1000 Ljubljana, Slovenia

5 Department of Applied Analysis and Computational Mathematics, ELTE TTK, Pázmány Péter sétány 1/C, 1117 Budapest, Hungary 
In the case of conservation law $(C L)$ studied in [6, Subsec. 4.1], a smaller set of weak solution should be considered. A function $v:[0, \infty) \rightarrow \mathrm{L}^{1}(\mathbb{R})$ is called an isentropic solution to $(C L)$, if it satisfies the initial condition $v(0)=h$ and the Kružkov condition (14) from [6] with the equality sign.

We now give the proper formulation of [6, Proposition 4.1] and its proof. Let

$$
\begin{aligned}
\mathcal{C}:= & \left\{h \in \mathrm{L}^{1}(\mathbb{R}) \mid(C L) \text { admits an isentropic solution } v \text { with } v(0)=h\right\} \\
& \cup\{-\infty\} .
\end{aligned}
$$

Proposition ([6, Proposition 4.1]) The semigroup $\mathcal{T}^{\mathrm{CL}}:=(T(t))_{t \geq 0}$, where $u(t, x)=$ $T(t) h(x)$ is the unique isentropic solution to $(C L)$ and where $T(t)(-\infty):=-\infty$, is a max-additive strongly continuous semigroup on $\mathcal{C}$.

Proof By [5, Theorem 1], $T(t) h_{1} \oplus T(t) h_{2}$ is an isentropic solution to (CL) for any $h_{1}, h_{2} \in \mathcal{C}$. Moreover, $\left.T(t) h_{1} \oplus T(t) h_{2}\right|_{t=0}=h_{1} \oplus h_{2}$, hence $T(t) h_{1} \oplus T(t) h_{2}$ is the isentropic solution to (CL) with initial condition $h=h_{1} \oplus h_{2} \in \mathcal{C}$. By uniqueness of the isentropic solutions, we obtain $T(t) h_{1} \oplus T(t) h_{2}=T(t)\left(h_{1} \oplus h_{2}\right)$.

Remark 1 The isentropic solutions to $(C L)$ often exist only on some limited time interval. However, our results also hold in this case considering the local time flows $(T(t))_{t \in[0, T]}$ defined on

$$
\begin{aligned}
\mathcal{C}_{T}:= & \left\{h \in \mathrm{L}^{1}(\mathbb{R}) \mid(C L) \text { admits an isentropic solution } v \text { on }[0, T]\right. \\
& \text { with } v(0)=h\} \cup\{-\infty\} .
\end{aligned}
$$

In the case of Hamilton-Jacobi equation $(H J)$ considered in [6, Subsec. 4.2], we should specify the choice of the generalized solutions and regularity assumptions. We assume the following for the function $f$ appearing in the problem $(H J)$.

1. For every $x \in \mathbb{R}^{n},\|x\|=1$, there exists the $\operatorname{limit}_{\lim } \downarrow \mathbf{l} f(x / r)$.

2. For any $(x, r),\left(x^{\prime}, r^{\prime}\right) \in \mathbb{R}^{n} \times \mathbb{R}_{+}$with $\|x\|^{2}+r^{2} \leq 1$ and $\left\|x^{\prime}\right\|^{2}+r^{\prime 2} \leq 1$,

$$
\left|r f(x / r)-r^{\prime} f\left(x^{\prime} / r^{\prime}\right)\right| \leq K\left(\left\|x-x^{\prime}\right\|^{2}+\left(r-r^{\prime}\right)^{2}\right)^{1 / 2}
$$

where $K>0$ is some constant.

3. The function $f$ is convex.

In the literature, there are many notions for generalized weak solutions to $(H J)$ : Crandall-Lions' viscosity solutions, minimax solutions by Subbotin, Maslov idempotent weak solutions, Kružkov generalized solutions. However, under our assumptions they all agree, see [7, Sec. 5], and [6, Proposition 4.2] holds as stated in the paper. For the proof, we refer directly to [7, Theorem 3.2] (note that there the min-plus terminology is used; therefore, the concavity instead of convexity of $f$ is assumed).

Remark 2 Stability of solutions of $(H J)$ (understood in the viscosity sense of Crandall-Lions) under the $\oplus$-operation is closely related to the properties of liminf of a sequence of viscosity solutions of first order Hamilton-Jacobi equations with 
convex Hamiltonians, see [2] and [1, Th. 2.1 and 2.3]. The fact that the maximum of two viscosity solutions is a viscosity sub-solution is classical, see [3]. Heuristically, the fact that the maximum of two viscosity solutions is also a viscosity super-solution stems from the semi-concavity property of viscosity solutions, see [1, p.1125].

Remark 3 We add a reference for yet another proof of the max-linearity of HamiltonJacobi-Bellman semigroup presented in Subsection 4.3. In [4], this fact is proved using a probabilistic approach.

Acknowledgements The first author thanks Guy Barles for fruitful discussions.

\section{References}

1. Barles, G.: Discontinuous viscosity solutions of first-order Hamilton-Jacobi equations: a guided visit. Nonlinear Anal. Theory Methods Appl. 20, 1123-1134 (1993)

2. Barron, E.N., Jensen, R.: Semicontinuous viscosity solutions for Hamilton-Jacobi equations with convex Hamiltonians. Commun. Partial Differ. Equ. 15, 1713-1742 (1990)

3. Crandall, M.G., Ishii, H., Lions, P.-L.: User's guide to viscosity solutions of second order partial differential equations. Bull. Am. Math. Soc. New Ser. 27, 1-67 (1992)

4. Del Moral, P., Doisy, M.: Maslov idempotent probability calculus. II. Theory Probab. Appl. 44, 319-332 (1999)

5. Korobkov, M.V., Panov, E.Y.: On the theory of isentropic solutions of quasilinear conservation laws. J. Math. Sci. 144, 3815-3824 (2007)

6. Kramar Fijavž, M., Peperko, A., Sikolya, E.: Semigroups of Max-Plus Linear Operators, Semigroup Forum. doi:10.1007/s00233-015-9761-x, to appear

7. Roublev, I.V.: On minimax and idempotent generalized weak solutions to the Hamilton-Jacobi equation. Idempotent Mathematics and Mathematical Physics, pp. 319-337. AMS, Providence (2005) 